\title{
OPTIMAL PROGRAMS AND THEIR PRICE CHARACTERIZATON IN A MULTISECTOR GROWTH MODEL WITH UNCERTAINTY
}

\author{
NIKOLAOS S. PAPAGEORGIOU
}

(Communicated by Joseph S. B. Mitchell)

\begin{abstract}
In this paper we examine a nonstationary multisector growth model with uncertainty in which future utilities are discounted. First we establish the existence of strongly optimal programs emanating from a given initial capital stock. Then we show that this optimal program $\hat{x}$ is sustained by a system of prices $\hat{p}$ so that the pair $[\hat{x}, \hat{p}]$ is competitive and a strong transversality condition holds. We also show that competitiveness and transversality imply optimality.
\end{abstract}

\section{INTRODUCTION}

In this paper we study a nonstationary, infinite horizon, multisector growth model with uncertainty and discounted future utilities. The uncertainty is present in both the technology multifunction and the utility. For this model first we establish the existence of a strongly optimal program, and then we show that such a program is characterized by a system of support prices. Our work here generalizes the deterministic work of Peleg and Ryder [9] and Weitzman [11] and the stochastic work of Zilha [13], who considered a less general, stationary model with finite-dimensional commodity space. In this paper we examine a nonstationary model, and for further mathematical generality we assume that the commodity space is infinite dimensional. In recent years many mathematical economists, in particular those working on equilibrium theory, have considered models with an infinite-dimensional commodity space (see the book of Aliprantis, Brown, and Burkinshaw [1] and the references therein). For such a general model we first show that there exists a feasible program (path) maximizing the discounted intertemporal utility, and then we show that this strongly optimal program is sustained by a system of prices, in the sense that

(i) at every state we have minimization of the cost among all programs producing no less future value;

(ii) at each stage we have maximization of the total utility (gain) measured as the sum of the direct utility and of the net profit from operating the particular

Received by the editors July 31, 1992 and, in revised form, December 14, 1992.

1991 Mathematics Subject Classification. Primary 90A20.

Key words and phrases. Multisector growth model, feasible program, discount factor, free disposability, intertemporal utility, support prices, transversality condition.

This research was supported by a grant from the Greek Ministry of Industry and Technology.

(C) 1994 American Mathematical Society $0002-9939 / 94 \$ 1.00+\$ .25$ per page 
program. Note that the net profit is the value of the output minus the cost of the input; and

(iii) the expected value of the optimal program goes to zero as time goes to infinity (transversality condition).

In establishing the existence of support prices, we use an induction argument and an extension of the Yosida-Hewitt decomposition theorem for the dual space $L^{\infty}(X)^{*}$, due to Levin [8].

The first important contributions in stochastic growth theory were made by Dynkin [15, 16] and Radner [18]. Soon after Evstigneev [17] and Taksar [19] made further contributions to the subject by considering more general models, similar to the one used here but with a finite-dimensional commodity space. A comprehensive introduction to the subject and additional references can be found in the book of Arkin and Evstigneev [14].

\section{THE MODEL}

Let $(\Omega, \Sigma, \mu)$ be a complete probability space. As always each $\omega \in \Omega$ represents a possible state of the environment, $\Sigma$ is the collection of all possible events, and $\mu(\cdot)$ is the probability distribution of the states. Our time horizon is $\mathbb{N}_{0}=\{0,1,2,3, \ldots\}$. So our model is discrete-time, with an infinite planning horizon. An infinite planning horizon is very appropriate in modelling dynamic economic systems. According to Arrow and Kurz [2], the infinite horizon is an idealization of the fundamental point that the consequences of investment are very long lived. Any short horizon requires some method of evaluating end-ofperiod capital stocks, and the only proper evaluation is their value in use in the future. The uncertainty about the states is described by an increasing family $\left\{\Sigma_{n}\right\}_{n \geq 0}$ of complete sub- $\sigma$-fields of $\Sigma$, such that $\bigvee_{n=0}^{\infty} \Sigma_{n}=\Sigma$. The sub- $\sigma$-field $\Sigma_{n}$ represents the information about the states, available up until time $n$. Our commodity space is a separable, reflexive Banach space $X$, partially ordered by a closed and convex cone $X_{+}$. We are also given a discount factor $\delta \in(0,1)$ with which we discount future utilities.

Before proceeding further with the detailed description of the model, let us comment on the infinite-dimensionality of the commodity space. Why a Banach space of goods? As we already mentioned in the introduction, mathematical economists working in equilibrium theory have been using an infinitedimensional commodity space for more than a decade now. A detailed exposition of the work in that direction can be found in the book of Aliprantis, Brown, and Burkinshaw [1]. One reason for considering an infinite-dimensional commodity space is that the same commodity at different times should be considered as different, thus leading to infinite dimensions. Also, some specific models (like the overlapping generations model, which is worked in detail in [1]) last indefinitely, so they are naturally infinite dimensional. In addition, we learn a lot about the conventional case when we try to extend it to an infinitedimensional context, where the mathematical framework does not offer all the technical conveniences of the standard case. Finally, an infinite-dimensional commodity space better depicts the situation in which we are dealing with a finite, yet very large, number of capital goods. Recall that in a parallel situation in equilibrium theory, starting with the fundamental work of Aumann, the very large number of agents was replaced by a continuum of agents. 
At each time instant $n \geq 1$, the technological possibilities of our economy are described by a multifunction $P_{n}: \Omega \times X_{+} \rightarrow 2^{X_{+}} \backslash\{\varnothing\}$ which is $\left(\Sigma_{n} \times B(X) \times\right.$ $B(X)$ )-graph-measurable; i.e., $\operatorname{Gr} P_{n} \in \Sigma_{n} \times B(X) \times B(X)$ (here $B(X)$ denotes the Borel $\sigma$-field of $X)$. The set $P_{n}(\omega)$ describes all possible transformations of capital stock at time $n$, when the state of the environment is $\omega \in \boldsymbol{\Omega}$. So $(x, y) \in P_{n}(\omega)$ means that when the state of the environment is $\omega \in \Omega$, with the technology available at time $n$, we can transform a capital input $x$ at time $n-1$ into a capital output $y$ at time $n$. Hence the preceding capital stocks do not uniquely determine the technologically feasible future stocks, so transition from one state of the economy to another is described by a multifunction. Note that uncertainty in the production process is manifested on the hypothesis that Gr $P_{n} \in \Sigma_{n} \times B(X) \times B(X)$.

At each stage $n \geq 1$, the utility (gain) of operating a production process $(x, y) \in X_{+} \times X_{+}$is expressed by a utility function $u_{n}: \Omega \times X_{+} \times X_{+} \rightarrow \mathbb{R}$ which is assumed to be $\left(\Sigma_{n} \times B(X) \times B(X)\right)$-measurable. At every stage the utility is discounted by a factor $\delta \in(0,1)$.

A "program" (policy, path) is a sequence $\left\{x_{n}\right\}_{n \geq 0}$ such that $x_{n} \in L^{\infty}\left(\Sigma_{n}, X\right)$. A program $\left\{x_{n}\right\}_{n \geq 0}$ is said to be "feasible" if $\left(x_{n}(\omega), x_{n+1}(\omega)\right) \in P_{n+1}(\omega) \mu$ a.e. for all $n \geq 0$. We will say that $\left\{x_{n}\right\}_{n \geq 0}$ is a feasible program starting from $\bar{x}_{0} \in L^{\infty}\left(\Sigma_{0}, X\right)$ if $x_{0}=\bar{x}_{0}$. We will denote the set of all feasible programs starting from $\bar{x}_{0}$ by $F\left(\bar{x}_{0}\right)$. Clearly then $F\left(\bar{x}_{0}\right) \subseteq \prod_{k \geq 0} L^{\infty}\left(\Sigma_{k}, X\right)$.

Given an initial capital stock $\bar{x}_{0} \in L^{\infty}\left(\Sigma_{0}, X\right), \bar{x}_{0} \geq 0$ (i.e., $\bar{x}_{0}(\omega) \geq 0$ $\mu$-a.e.), our goal is to find a program in $F\left(\bar{x}_{0}\right)$ which maximizes the expected intertemporal discounted utility $U(\hat{x})=\sum_{k=0}^{\infty} \delta^{k+1} J_{k+1}\left(x_{k}, x_{k+1}\right)$ with $\hat{x}=$ $\left\{x_{n}\right\}_{n \geq 0}$ and $J_{k+1}(v, w)=\int_{\Omega} u_{k+1}(\omega, v(\omega), w(\omega)) d \mu(\omega)$ for all $(v, w) \in$ $L^{\infty}\left(\Sigma_{k}, X\right) \times L^{\infty}\left(\Sigma_{k+1}, X\right), v, w \geq 0$.

The detailed mathematical hypotheses on the data of our model are:

$H(P) . P_{k}: \Omega \rightarrow 2^{X_{+} \times X_{+}} \backslash\{\varnothing\}, k \geq 1$, is a multifunction with closed and convex values such that

(1) $\mathrm{Gr} P_{k} \in \Sigma_{k} \times B(X) \times B(X)$;

(2) for every $\omega \in \Omega,(0,0) \in P_{k}(\omega)$ and if $(0, y) \in P_{k}(\omega)$, then $y=0$;

(3) for every $\omega \in \Omega$, if $(x, y) \in P_{k}(\omega), x \leq x^{\prime}, y^{\prime} \leq y$, then $\left(x^{\prime}, y^{\prime}\right) \in$ $P_{k}(\omega) ;$ and

(4) there exists $M>0$ such that for all $\omega \in \Omega$, if $(x, y) \in P_{k}(\omega)$ and $\|x\| \geq M$, then $\|y\| \leq\|x\|$.

As we already mentioned, hypothesis $H(P)(1)$ expresses the uncertainty involved in the production process. The closedness and convexity of the values of $P_{k}(\cdot)$ and hypotheses $H(P)(2)-(4)$ are very common in deterministic multisector growth models (see, for example, Takayama [10]). The closedness requirement for the sets $P_{k}(\omega)$ is primarily a mathematical one, which is thought consistent with economic principles, while the convexity hypothesis follows from the well-known "law of diminishing returns to scale". Hypothesis $H(P)(2)$ says that inaction is always an option and that there is no free production ("no horn of plenty"). Hypothesis $H(P)(3)$ is the usual free disposability hypothesis. Finally, hypothesis $H(P)(4)$ means that when capital exists in sufficiently large quantities, there is loss due to depreciation. This hypothesis will guarantee for us the boundedness of the elements in $F\left(\bar{x}_{0}\right)$. 
$H(u) . \quad u_{k}: \Omega \times X_{+} \times X_{+} \rightarrow \mathbb{R}, k \geq 1$, is a function such that

(1) $(\omega, x, y) \rightarrow u_{k}(\omega, x, y)$ is $\left(\Sigma_{k} \times B(X) \times B(X)\right)$-measurable;

(2) $(x, y) \rightarrow u_{k}(\omega, x, y)$ is concave and u.s.c; and

(3) $\left|u_{k}(\omega, x, y)\right| \leq \varphi_{k}(\omega,\|x\|,\|y\|)$ with $\varphi_{k}(\omega, \cdot, \cdot)$ increasing and $\sup _{k \geq 1}\left\|\varphi_{k}(\cdot, v, v)\right\|_{1}<\infty$.

Again these are very common hypotheses about the utility function in multisector growth models (see Takayama [10] and Arkin and Evstigneev [14]).

By a "price system" we mean a sequence $\hat{p}=\left\{p_{n}\right\}_{n \geq 0}, p_{n} \in L^{1}\left(\Sigma_{n}, X^{*}\right)$, $p_{n} \geq 0$. Recall that the dual space $L^{\infty}\left(\Sigma_{n}, X\right)^{*}$ is much bigger than $L^{1}\left(\Sigma_{n}, X^{*}\right)$ and that the two spaces are related via the Yosida-Hewitt decomposition theorem [12], which was extended to Lebesgue-Bochner spaces by Levin [8].

A functional $p \in L^{\infty}\left(\Sigma_{n}, X\right)^{*}$ is said to be absolutely continuous with respect to $\mu(\cdot)$ if there exists $q \in L^{1}\left(\Sigma_{n}, X^{*}\right)$ such that

$$
p(x)=\int_{\Omega}(q(\omega), x(\omega)) d \mu(\omega)
$$

for all $x \in L^{\infty}\left(\Sigma_{n}, X\right)$. For economy in the notation, in the sequel by $\langle\cdot, \cdot\rangle$ we will denote the duality brackets for the pair $\left(L^{1}\left(\Sigma_{n}, X^{*}\right), L^{\infty}\left(\Sigma_{n}, X\right)\right)$; i.e., $\langle q, x\rangle=\int_{\Omega}(q(\omega), x(\omega)) d \mu(\omega)$ with $q \in L^{1}\left(\Sigma_{n}, X^{*}\right), x \in L^{\infty}\left(\Sigma_{n}, X\right)$. Also we will identify an absolutely continuous functional $p$ with the function $q \in$ $L^{1}\left(\Sigma_{n}, X^{*}\right)$.

A functional $p \in L^{\infty}\left(\Sigma_{n}, X\right)^{*}$ is said to be singular with respect to $\mu(\cdot)$ if there exists a sequence $\left\{B_{m}\right\}_{m \geq 1} \subseteq \Sigma_{n}$ such that

(i) $\boldsymbol{B}_{m+1} \subseteq \boldsymbol{B}_{\boldsymbol{m}}$ for all $m \geq 1$,

(ii) $\mu\left(B_{m}\right) \rightarrow 0$ as $m \rightarrow \infty$; and

(iii) $p(x)=p\left(\chi_{B_{m}} x\right)$ for all $m \geq 1$ and all $x \in L^{\infty}\left(\Sigma_{n}, X\right)$; i.e., the sets $B_{m}$ support the functional $p$.

There is the following decomposition theorem for $L^{\infty}\left(\Sigma_{n}, X\right)^{*}$. It was first proved for $\mathbb{R}$-valued functions by Yosida and Hewitt [12] and extended to Lebesgue-Bochner spaces by Levin [8].

Proposition 2.1. Every functional $p \in L^{\infty}\left(\Sigma_{n}, X\right)^{*}$ admits a unique decomposition $p=p^{a}+p^{s}$, where $p^{a}(\cdot)$ is absolutely continuous and $p^{s}(\cdot)$ is singular with respect to $\mu(\cdot)$. Furthermore, $\|p\|=\left\|p^{a}\right\|+\left\|p^{s}\right\|$.

Remark. In fact, the result is true even if $X$ is not separable or reflexive. In this case then, $q \in L^{1}\left(\Sigma_{n}, X_{w^{*}}^{*}\right)$; i.e., for every $x \in X, \omega \rightarrow(q(\omega), x)$ is measurable ( $w^{*}$-measurability) and $\|q(\cdot)\| \in L^{1}\left(\Sigma_{n}\right)_{+}$. For details, we refer to Levin [8].

The Mackey topology $\tau$ on $L^{\infty}\left(\Sigma_{n}, X\right)$ induced by the pair $\left[L^{\infty}\left(\Sigma_{n}, X\right)\right.$, $\left.L^{1}\left(\Sigma_{n}, X^{*}\right)\right]$ is by definition the topology of uniform convergence on weakly compact, convex subsets of $L^{1}\left(\Sigma_{n}, X^{*}\right)$. In what follows by $\stackrel{\mu}{\rightarrow}$ we will denote the convergence in $\mu$-measure.

Proposition 2.2. If $\left\{g_{m}, g\right\}_{m \geq 1} \subseteq L^{\infty}\left(\Sigma_{n}, X\right)\left\|g_{m}\right\|_{\infty} \leq \eta$, and $g_{m} \stackrel{\mu}{\rightarrow} g$ as $m \rightarrow \infty$, then $g_{m} \stackrel{\tau}{\rightarrow} g$ in $L^{\infty}\left(\Sigma_{n}, X\right)$.

Proof. Let $W$ be a nonempty, weakly compact and convex subset of $L^{1}\left(\Sigma_{n}, X^{*}\right)$. We need to show that $\sup _{w \in W}\left|\left\langle w, g_{m}-g\right\rangle\right| \rightarrow 0$ as $m \rightarrow \infty$. To simplify our proof, we can assume without any loss of generality that $g=0$. 
Also, since $W$ is weakly compact from Theorem 4, p. 104, of Diestel and Uhl [5], we know that $W$ is uniformly integrable. Therefore, the set $\{v=$ $\left.\|h\| \cdot\|w\| \in L^{1}\left(\Sigma_{n}\right):\|h\|_{\infty} \leq \eta, w \in W\right\}$ is uniformly integrable in $L^{1}\left(\Sigma_{n}\right)$. Thus given $\varepsilon>0$, we can find $\varphi \in L^{1}\left(\Sigma_{n}\right)_{+}, \varphi>0$, such that for all $\|h\|_{\infty} \leq \eta$ and all $w \in W$ we have

$$
\int_{\{\|h\| \cdot\|w\| \geq \varphi\}}\|h(\omega)\| \cdot\|w(\omega)\| d \mu(\omega) \leq \varepsilon .
$$

So it is clear that we have to show

$$
\lim _{m \rightarrow \infty} \sup _{w \in W} \int_{\left\{\left\|g_{m}\right\| \cdot\|w\|<\varphi\right\}}\left\|g_{m}(\omega)\right\| \cdot\|w(\omega)\| d \mu(\omega)=0
$$

Since $W$ is bounded, we may assume without any loss of generality that $|W|=\sup \left\{\|w\|_{1}: w \in W\right\} \leq 1$. Note that $\{\omega \in \Omega: \varphi(\omega)=0\}=$ $\bigcap_{\lambda>0}\{\omega \in \Omega: \varphi(\omega)<\lambda\}$. Since $\varphi(\omega)>0 \mu$-a.e. and using the absolute continuity of the Lebesgue integral, we know that we can find $\theta>0$ such that $\int_{\{\varphi<\theta\}} \varphi(\omega) d \mu(\omega) \leq \varepsilon$. Let $\delta>0$ be such that if $A \in \Sigma_{n}$ with $\mu(A) \leq \delta$, then $\int_{A} \varphi(\omega) d \mu(\omega) \leq \varepsilon$. Let $0<\gamma<\min (\varepsilon, \delta)$. Since by hypothesis $g_{m} \stackrel{\mu}{\rightarrow} g=0$, there exists $m_{0} \geq 1$ such that for $m \geq m_{0}$ we have $\mu\left\{\omega \in \Omega: \varphi(\omega) \geq \theta,\left\|g_{m}(\omega)\right\| \geq \gamma\right\} \leq \gamma$. So for $m \geq m_{0}$ and all $w \in W$ we have

$$
\begin{aligned}
& \int_{\left\{\left\|g_{m}\right\| \cdot\|w\|<\varphi\right\} \cap\{\varphi \geq \theta\} \cap\left\{\left\|g_{m}\right\| \geq \gamma\right\}}\left\|g_{m}(\omega)\right\| \cdot\|w(\omega)\| d \mu(\omega) \\
& \left.\leq \int_{\{\varphi \geq \theta\} \cap\left\{\left\|g_{m}\right\| \geq \gamma\right\}} \varphi(\omega) d \mu(\omega) \leq \varepsilon \quad \text { (recall the choice of } \gamma>0\right) .
\end{aligned}
$$

Also for all $m \geq m_{0}$ and for all $w \in W$ we have

$$
\int_{\left\{\left\|g_{m}\right\| \leq \gamma\right\}}\left\|g_{m}(\omega)\right\| \cdot\|w(\omega)\| d \mu(\omega) \leq \gamma
$$

(recall that we have assumed that $|W| \leq 1$ ).

So we get that for all $m \geq m_{0}$ and all $w \in W$ we have

$$
\begin{aligned}
\int_{\left\{\left\|g_{m}\right\| \cdot\|w\|<\varphi\right\}}\left\|g_{m}(\omega)\right\| \cdot\|w(\omega)\| d \mu(\omega) \\
=\int_{\left\{\left\|g_{m}\right\| \cdot\|w\|<\varphi\right\} \cap\{\varphi \geq \theta\} \cap\left\{\left\|g_{m}\right\| \geq \gamma\right\}}\left\|g_{m}(\omega)\right\| \cdot\|w(\omega)\| d \mu(\omega) \\
\quad+\int_{\left\{\left\|g_{m}\right\| \cdot\|w\|<\varphi\right\} \cap\left[\{\varphi<\theta\} \cup\left\{\left\|g_{m}\right\|<\gamma\right\}\right]}\left\|g_{m}(\omega)\right\| \cdot\|w(\omega)\| d \mu(\omega) \\
\leq \varepsilon+\varepsilon+\varepsilon=3 \varepsilon \quad \text { Q.E.D. }
\end{aligned}
$$

\section{EXISTENCE OF OPTIMAL PROGRAMS}

In this section we show that given an initial capital stock $\bar{x}_{0} \in L^{1}\left(\Sigma_{0}, X\right)$, $\bar{x}_{0} \geq 0$, we can find a program $\hat{x} \in F\left(\bar{x}_{0}\right)$ which maximizes the intertemporal utility $U(\hat{x})$. In what follows by $w_{\text {seq }}$ we will denote the topology on 
$L^{1}\left(\Sigma_{n}, X\right), n \geq 1$, whose closed sets are the sequentially weakly closed sets (see Buttazzo [4, Proposition 1.1.5, p. 9] and Dolcher [6]).

Theorem 3.1. If hypotheses $H(P)$ and $H(u)$ hold and $\bar{x}_{0} \in L^{\infty}\left(\Sigma_{0}, X\right), \bar{x}_{0} \geq$ 0 , then there exists an optimal program in $F\left(\bar{x}_{0}\right)$.

Proof. We will start by showing that if $\hat{x} \in F\left(\bar{x}_{0}\right)$, then, for all $n \geq 0,\left\|x_{n}\right\|_{\infty} \leq$ $M_{1}$ with $M_{1}=\max \left[\left\|\bar{x}_{0}\right\|_{\infty}, M\right]$. To this end we will show that if $n \geq 1$ and $(x, y) \in P_{n}(\omega)$, then $\|y\| \leq \max [\|x\|, M]$. Suppose not. Then according to hypothesis $H(P)(4)$ there exists $(x, y) \in P_{n}(\omega)$ such that $\|x\| \leq M$ and $\|y\|>M$. Clearly, because of hypothesis $H(P)(2), x \neq 0$. Set $x^{\prime}=M \frac{x}{\|x\|}$. Then we have $x^{\prime}-x=\left(\frac{M}{\|x\|}-1\right) x \geq 0$, so by the free disposability hypothesis we have $\left(x^{\prime}, y\right) \in P_{n}(\omega)$. But note that $\left\|x^{\prime}\right\|=M$. So by hypothesis $H(P)(4)$ we must have $\|y\| \leq\left\|x^{\prime}\right\|=M$, a contradiction. So indeed we have that $\|y\| \leq \max [\|x\|, M]$, and thus for every $\hat{x} \in F\left(\bar{x}_{0}\right)$ we have that $\left\|x_{n}\right\|_{\infty} \leq$ $M_{1}=\max \left[\left\|\bar{x}_{0}\right\|_{\infty}, M\right], n \geq 0$.

Let $B_{k}=\left\{h \in L^{1}\left(\Sigma_{k}, X\right):\|h\|_{1} \leq M_{1}\right\}$. From the Dunford-Pettis theorem (see Diestel and Uhl [5, Theorem 1, p. 101]) and the Eberlein-Smulian

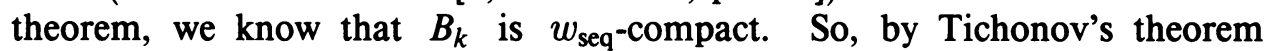
$\prod_{k \geq 0} B_{k}$ is compact for the $w_{\text {seq }}$-product topology. Since $F\left(\bar{x}_{0}\right) \subseteq \prod_{k \geq 0} B_{k}$ and clearly, by hypothesis $H(P), F\left(\bar{x}_{0}\right)$ is a nonempty closed and convex subset of $\prod_{k \geq 0} L^{1}\left(\Sigma_{k}, X\right)$, we have that $F\left(\bar{x}_{0}\right)$ is compact for the $w_{\text {seq }}$-product topology.

Next let $u_{n}(\omega, x, y)=-\infty$ if $(\omega, x, y) \notin \Omega \times X_{+} \times X_{+}$; i.e., we define a utility function $\hat{u}_{n}(\omega, x, y)$ by setting

$$
\hat{u}_{n}(\omega, x, y)=u_{n}(\omega, x, y)+\delta_{\mathrm{Gr} P_{n}}(\omega, x, y),
$$

where $\delta_{\mathrm{Gr} P_{n}}(\omega, x, y)=0$ if $(\omega, x, y) \in \mathrm{Gr} P_{n}$ and $\delta_{\mathrm{Gr} P_{n}}(\omega, x, y)=-\infty$ if $(\omega, x, y) \notin \operatorname{Gr} P_{n}$. Using hypothesis $H(P)$, we can easily check that this extended utility function still satisfies hypothesis $H(u)$, but now as an $(\mathbb{R}=$ $\mathbb{R} \cup\{-\infty\}$ )-valued function.

We claim that $\hat{U}: \prod_{n \geq 0} L^{1}\left(\Sigma_{n}, X\right) \rightarrow \overline{\mathbb{R}}=\mathbb{R} \cup\{-\infty\}$ is $w_{\text {seq }}$-u.s.c. on $F\left(\bar{x}_{0}\right)$, where

$$
\hat{U}(\hat{x})=\sum_{n=0}^{\infty} \delta^{n+1} \hat{J}_{n+1}\left(x_{n}, x_{n+1}\right)
$$

and

$$
\hat{J}_{n+1}(x, y)=\int_{\Omega} \hat{u}(\omega, x(\omega), y(\omega)) d \mu(\omega)
$$

for all $x \in L^{1}\left(\Sigma_{n}, X\right), y \in L^{1}\left(\Sigma_{n+1}, X\right)$. Because of hypothesis $H(u)$ and the definition of $\hat{u}(\omega, x, y), \quad \hat{J}_{n+1}(\cdot, \cdot)$ is concave and u.s.c. on $L^{1}\left(\Sigma_{n}, X\right) \times$ $L^{1}\left(\Sigma_{n+1}, X\right)$, so $\hat{J}_{n+1}(\cdot, \cdot)$ is weakly-u.s.c., in particular then, $w_{\text {seq }}$-u.s.c. (recall that the $w_{\text {seq }}$-topology is in general stronger than the weak topology). Now let $\hat{x}^{m} \in F\left(\bar{x}_{0}\right), \hat{x}^{m} \stackrel{w_{\text {seq }}}{\rightarrow} \hat{x} \in F\left(\bar{x}_{0}\right)$. Then for every $n \geq 0$ we have $x_{n}^{m} \stackrel{w_{\text {sog }}}{\rightarrow} x_{n}$ as $m \rightarrow \infty$ in $L^{1}\left(\Sigma_{n}, X\right)$, so

$$
\varlimsup_{m \rightarrow \infty} \hat{J}_{n+1}\left(x_{n}^{m}, x_{n+1}^{m}\right) \leq \hat{J}_{n+1}\left(x_{n}, x_{n+1}\right) .
$$


So if $\hat{U}_{N}(\hat{v})=\sum_{n=0}^{N} \delta^{n+1} \hat{J}_{n+1}\left(v_{n}, v_{n+1}\right), \hat{v} \in \prod_{n \geq 0} L^{1}\left(\Sigma_{n}, X\right)$, we get

$$
\begin{aligned}
\varlimsup_{m \rightarrow \infty} \hat{U}_{N}\left(\hat{x}^{m}\right) & =\varlimsup_{m \rightarrow \infty} \sum_{n=0}^{N} \delta^{n+1} \hat{J}_{n+1}\left(x_{n}^{m}, x_{n+1}^{m}\right) \\
& \leq \sum_{n=0}^{N} \delta^{n+1} \varlimsup_{m \rightarrow \infty} \hat{J}_{n+1}\left(x_{n}^{m}, x_{n+1}^{m}\right) \\
& \leq \sum_{n=0}^{N} \delta^{n+1} \hat{J}_{n+1}\left(x_{n}, x_{n+1}\right)=\hat{U}_{N}(\hat{x}) .
\end{aligned}
$$

On the other hand, since $\hat{x} \in F\left(x_{0}\right)$, we have

$$
\left|\hat{U}(\hat{x})-U_{N}(\hat{x})\right|=\left|\sum_{n=N+1}^{\infty} \delta^{n+1} \hat{J}_{n+1}\left(x_{n}, x_{n+1}\right)\right| \leq \sum_{n=N+1}^{\infty} \delta^{n+1} \beta,
$$

where $\beta=\sup _{n \geq 1}\left\|\varphi_{n}\left(\cdot, M_{1}, M_{1}\right)\right\|_{1}<\infty$; see hypothesis $H(u)(3)$.

So $\hat{U}_{N}(\hat{x}) \rightarrow U(\hat{x})$ as $N \rightarrow \infty$. Then invoking [3, Corollary 1.16, p. 33], we get that there exists a map $m \rightarrow N(m)$ increasing to infinity such that

$$
\varlimsup_{m \rightarrow \infty} \hat{U}_{N(m)}\left(\hat{x}^{m}\right) \leq \varlimsup_{N \rightarrow \infty}\left(\varlimsup_{m \rightarrow \infty} \hat{U}_{N(m)}\left(\hat{x}^{m}\right)\right) \leq \varlimsup_{N \rightarrow \infty} \hat{U}_{N}(\hat{x})=\hat{U}(\hat{x}) .
$$

Hence we have

$$
\begin{aligned}
& \hat{U}\left(\hat{x}^{m}\right)-\hat{U}(\hat{x})=\left[\hat{U}\left(\hat{x}^{m}\right)-\hat{U}_{N(m)}\left(\hat{x}^{m}\right)\right]+\left[\hat{U}_{N(m)}\left(\hat{x}^{m}\right)-\hat{U}(\hat{x})\right] \\
& \leq \sum_{n=N(m)+1}^{\infty} \delta^{n+1} \beta+\left[\hat{U}_{N(m)}\left(\hat{x}^{m}\right)-\hat{U}(\hat{x})\right] \\
& \Rightarrow \varlimsup_{m \rightarrow \infty}\left(\hat{U}\left(\hat{x}^{m}\right)-\hat{U}(\hat{x})\right) \\
& \leq \varlimsup_{m \rightarrow \infty} \sum_{n=N(m)+1}^{\infty} \delta^{n+1} \beta+\varlimsup_{m \rightarrow \infty}\left[\hat{U}_{N(m)}\left(\hat{x}^{m}\right)-\hat{U}(\hat{x})\right] \leq 0 \\
& \Rightarrow \varlimsup_{\lim } \hat{U}\left(\hat{x}^{m}\right) \leq \hat{U}(\hat{x}) .
\end{aligned}
$$

So we have checked that $\hat{U}(\cdot)$ is $w_{\text {seq }}$-u.s.c. on $F\left(\bar{x}_{0}\right)$. Then from Weierstrass's theorem we know that there exists $x^{*} \in H\left(\bar{x}_{0}\right)$ such that

$$
\sup \left[\hat{U}(\hat{x}): \hat{x} \in F\left(\bar{x}_{0}\right)\right]=\sup \left[U(\hat{x}): \hat{x} \in F\left(\bar{x}_{0}\right)\right]=U\left(x^{*}\right)
$$

$\Rightarrow \hat{x}^{*}=\left\{x_{n}^{*}\right\}_{n \geq 0}$ is the desired optimal program emanating from $x_{0}$. Q.E.D.

\section{SUPPORT PRICES}

In this section we establish the existence of a price system which sustains the optimal growth path, whose existence was proved in Theorem 3.1. In this section we will assume that int $X_{+} \neq \varnothing$.

So let $f \in L^{\infty}\left(\Sigma_{n}, X\right), n \geq 0$, and define

$$
C_{n}(f)=\left\{\hat{h}=\left\{h_{k}\right\}_{k \geq n}: h_{n}=f \text { and }\left(h_{k(\omega)}, h_{k+1}(\omega)\right) \in P_{k+1}(\omega) \mu \text {-a.e. }\right\} \text {. }
$$


Using this set we can define the following value (Bellman) function:

$$
\begin{aligned}
V_{n}(f) & =\sup \left[\sum_{k=n}^{\infty} \delta^{k+1} \hat{J}_{k+1}\left(h_{k}, h_{k+1}\right): \hat{h} \in C_{n}(f)\right] \\
& =\sup \left[\sum_{k=n}^{\infty} \delta^{k+1} J_{k+1}\left(h_{k}, h_{k+1}\right): \hat{h} \in C_{n}(f)\right] .
\end{aligned}
$$

We know that this function satisfies the following functional equation ("dynamic programming equation"):

$$
\begin{aligned}
V_{n}(f)=\sup \left[\delta^{n+1} J_{n+1}(f, g)+V_{n+1}(g):\right. & g \in L^{\infty}\left(\Sigma_{n+1}, X\right), \\
& \left.(f(\omega), g(\omega)) \in P_{n+1}(\omega) \mu \text {-a.e. }\right] .
\end{aligned}
$$

Furthermore, it is easy to see that if $\hat{x}^{*}=\left\{x_{n}^{*}\right\}_{n \geq 0}$ is an optimal program in $F\left(\bar{x}_{0}\right)$, then for all $n \geq 0$ we have

$$
V_{n}\left(x_{n}^{*}\right)=\delta^{n+1} J_{n+1}\left(x_{n}^{*}, x_{n+1}^{*}\right)+V_{n+1}\left(x_{n+1}^{*}\right) .
$$

In what follows, by $S_{n+1}$ we denote the nonempty, closed and convex subset of $L^{\infty}\left(\Sigma_{n}, X\right) \times L^{\infty}\left(\Sigma_{n+1}, X\right)$ defined by

$S_{n+1}=\left\{(f, g) \in L^{\infty}\left(\Sigma_{n}, X\right) \times L^{\infty}\left(\Sigma_{n+1}, X\right):(f(\omega), g(\omega)) \in P_{n+1}(\omega) \mu\right.$-a.e. $\}$.

Observe that because of hypothesis $H(u)$ the value function $V_{n}(\cdot)$ is concave on $L^{\infty}\left(\Sigma_{n}, X\right)$. For any $z \in L^{\infty}\left(\Sigma_{n}, X\right)$ its subdifferential $\partial V_{n}(x)$ is defined by

$\partial V_{n}(z)=\left\{p \in L^{\infty}\left(\Sigma_{n}, X\right)^{*}: V_{n}(y)-V_{n}(z) \leq p(y-z)\right.$ for all $\left.y \in L^{\infty}\left(\Sigma_{n}, X\right)\right\}$.

We will need the following hypotheses:

$H(u)_{1}, u_{k}: \Omega \times X_{+} \times X_{+} \rightarrow \mathbb{R}, k \geq 1$, is an integrand such that

(1) $\omega \rightarrow u_{k}(\omega, x, y)$ is $\Sigma_{k}$-measurable;

(2) $(x, y) \rightarrow u_{k}(\omega, x, y)$ is concave and continuous;

(3) $u_{k}(\omega, \cdot, y)$ is increasing, while $u_{k}(\omega, x, \cdot)$ is decreasing; and

(4) $\left|u_{k}(\omega, x, y)\right| \leq \varphi_{k}(\omega,\|x\|,\|y\|) \mu$-a.e. with $\varphi_{k}(\omega, \cdot, \cdot)$ increasing and $\sup _{k \geq 1}\left\|\varphi_{k}\left(\cdot, M^{\prime}, M^{\prime}\right)\right\|_{1}<\infty$.

$H_{0}$. For every $n \geq 0, V_{n}(\cdot)$ is continuous at some point in $L^{\infty}\left(\Sigma_{n}, X\right)$, and $\partial V_{0}\left(\bar{x}_{0}\right) \neq \varnothing$.

Remark. The first part of hypothesis $H_{0}$ is equivalent to saying that the value function is bounded from below in the neighborhood of a point (see Ioffe and Tichomirov [7, Theorem 1, p. 170]). If the technology multifunction admits a feasible program which is interior uniformly for almost all $\omega \in \Omega$ (i.e., $\left(v_{n}(\omega), v_{n+1}(\omega)\right)+B_{\varepsilon_{n+1}} \subseteq P_{n+1}(\omega) \mu$-a.e. with $B_{\varepsilon_{n+1}}=\{(v, w) \in X \times X:\|v\|+$ $\|w\| \leq \varepsilon\})$, then we can check that $V_{n}(\cdot)$ is bounded below in a neighborhood of $x_{n}(\cdot)$, so hypothesis $H_{0}$ is satisfied. Furthermore, if for this interior program we have that $v_{0}=\bar{x}_{0}$, then $\partial V_{0}\left(\bar{x}_{0}\right) \neq \varnothing$ (see [7, Proposition 3, p. 199]).

Theorem 4.1. If hypotheses $H(P), H(u)_{1}$, and $H_{0}$ hold, $\bar{x}_{0} \in L^{\infty}\left(\Sigma_{0}, X\right)$, $\bar{x}_{0} \geq 0$, and $\hat{x}^{*} \in F\left(\bar{x}_{0}\right)$ is an optimal program, then there exists a system of prices $p_{n} \in L^{1}\left(\Sigma_{n}, X\right), p_{n} \geq 0$, such that

(i) $V_{n}(f)-V_{n}\left(x_{n}^{*}\right) \leq\left\langle p_{n}, f-x_{n}^{*}\right\rangle$ for all $f \in L^{\infty}\left(\Sigma_{n}, X\right)$; 
(ii) $\delta^{n+1} J_{n+1}(f, g)-\left\langle p_{n}, f\right\rangle+\left\langle p_{n+1}, g\right\rangle \leq \delta^{n+1} J_{n+1}\left(x_{n}^{*}, x_{n+1}^{*}\right)-\left\langle p_{n}, x_{n}^{*}\right\rangle+$ $\left\langle p_{n+1}, x_{n+1}^{*}\right\rangle$ for all $(f, g) \in S_{n+1} ;$ and

(iii) $\lim _{n \rightarrow \infty}\left\langle p_{n}, x_{n}^{*}\right\rangle=0$.

Proof. Let $\eta_{1}^{n}: L^{\infty}\left(\Sigma_{n}, X\right) \times L^{\infty}\left(\Sigma_{n+1}, X\right) \rightarrow L^{\infty}\left(\Sigma_{n}, X\right)$ and $\eta_{2}^{n}: L^{\infty}\left(\Sigma_{n}, X\right)$ $\times L^{\infty}\left(\Sigma_{n+1}, X\right) \rightarrow L^{\infty}\left(\Sigma_{n+1}, X\right)$ be defined by $\eta_{1}^{n}(f, g)=f$ and $\eta_{2}^{n}(f, g)=$ $g$, respectively. Then $\eta_{1}^{n^{*}}: L^{\infty}\left(\Sigma_{n}, X\right)^{*} \rightarrow L^{\infty}\left(\Sigma_{n}, X\right)^{*} \times L^{\infty}\left(\Sigma_{n+1}, X\right)^{*}$ and $\eta_{2}^{n^{*}}: L^{\infty}\left(\Sigma_{n+1}, X\right)^{*} \rightarrow L^{\infty}\left(\Sigma_{n}, X\right)^{*} \times L^{\infty}\left(\Sigma_{n+1}, X\right)^{*}$ are defined by $\eta_{1}^{n^{*}}(v)=$ $(v, 0)$ and $\eta_{2}^{n^{*}}(w)=(0, w)$, respectively.

Using the maps $\eta_{1}^{n}$ and $\eta_{2}^{n}$, we define

$$
\xi_{1}^{n}=V_{n} o \eta_{1}^{n} \text { and } \xi_{2}^{n}=\delta^{n+1} \hat{J}_{n+1}+V_{n+1} o \eta_{2}^{n}+\delta_{S_{n+1}}
$$

where recall that $\delta_{S_{n+1}}(f, g)=0$ if $(f, g) \in S_{n+1}$ and $\delta_{S_{n+1}}(f, g)=-\infty$ if $(f, g) \notin S_{n+1}$ (indicator function for the set $\left.S_{n+1}\right)$. Both functions are clearly concave.

From the dynamic programming equation, we have for all $(f, g) \in$ $L^{\infty}\left(\Sigma_{n}, X\right) \times L^{\infty}\left(\Sigma_{n+1}, X\right)$ that

$$
\xi_{2}^{n}(f, g) \leq \xi_{1}^{n}(f, g) .
$$

Furthermore, since by hypothesis $\hat{x}^{*} \in F\left(\bar{x}_{0}\right)$ is optimal, we have

$$
\xi_{2}^{n}\left(x_{n}^{*}, x_{n+1}^{*}\right)=\xi_{1}^{n}\left(x_{n}^{*}, x_{n+1}^{*}\right) .
$$

Hence directly from the definition of the concave subdifferential we get

$$
\partial \xi_{1}^{n}\left(x_{n}^{*}, x_{n+1}^{*}\right) \subseteq \partial \xi_{2}^{n}\left(x_{n}^{*}, x_{n+1}^{*}\right) .
$$

Because of hypothesis $H_{0}$, we can use [7, Theorem 2, p. 201] and get

$$
\partial \xi_{1}^{n}\left(x_{n}^{*}, x_{n+1}^{*}\right)=\eta_{1}^{n^{*}} \partial V_{n}\left(x_{n}^{*}\right) \text {. }
$$

Also note that since we have assumed in this section that int $X_{+} \neq \varnothing$, it follows that int $L^{\infty}\left(\Sigma_{n}, X\right)_{+} \neq \varnothing$ for all $n \geq 0$, and, because of hypothesis $H(u)_{1}$, $\hat{J}_{n+1}(\cdot, \cdot)$ is continuous on int $L^{\infty}\left(\Sigma_{n}, X\right)_{+} \times$int $L^{\infty}\left(\Sigma_{n}, X\right)_{+} \cdot$. Combining this with hypothesis $H_{0}$ and [7, Theorem 1, p. 200], we get

$$
\begin{aligned}
\partial \xi_{2}^{n}\left(x_{n}^{*}, x_{n+1}^{*}\right)= & \partial\left[\delta^{n+1} \hat{J}_{n+1}+V_{n+1} o \eta_{2}^{n}+\delta_{S_{n+1}}\right]\left(x_{n}^{*}, x_{n+1}^{*}\right) \\
= & \delta^{n+1} \partial \hat{J}_{n+1}\left(x_{n}^{*}, x_{n+1}^{*}\right)+\partial\left(V_{n+1} o \eta_{2}^{n}\right)\left(x_{n}^{*}, x_{n+1}^{*}\right) \\
& +\partial \delta_{S_{n+1}}\left(x_{n}^{*}, x_{n+1}^{*}\right) .
\end{aligned}
$$

A new application of [7, Theorem 2, p. 201] on the second term in the above sum finally gives us

$$
\partial \xi_{2}^{n}\left(x_{n}^{*}, x_{n+1}^{*}\right)=\delta^{n+1} \partial \hat{J}_{n+1}\left(x_{n}^{*}, x_{n+1}^{*}\right)+\eta_{2}^{n^{*}} \partial V_{n+1}\left(x_{n+1}^{*}\right)+\partial \delta_{S_{n+1}}\left(x_{n}^{*}, x_{n+1}^{*}\right) .
$$

So if $p_{n} \in \partial V_{n}\left(x_{n}^{0}\right)$, then we can find “ $\left(z_{n}, z_{n+1}\right) \in \partial \hat{J}_{n+1}\left(x_{n}^{*}, x_{n+1}^{*}\right), p_{n+1} \in$ $\partial V_{n+1}\left(x_{n+1}^{*}\right),\left(y_{n}, y_{n+1}\right) \in \partial \delta_{S_{n+1}}\left(x_{n}^{*}, x_{n+1}^{*}\right)$ " such that

$$
\begin{aligned}
& \left(p_{n}, 0\right)=\left(\delta^{n+1} z_{n}, \delta^{n+1} z_{n+1}\right)+\left(0, p_{n+1}\right)+\left(y_{n}, y_{n+1}\right) \\
& \Rightarrow p_{n}=\delta^{n+1} z_{n}+y_{n} \text { and }-p_{n+1}=\delta^{n+1} z_{n+1}+y_{n+1} .
\end{aligned}
$$

First we will show that $p_{n+1}^{a} \in \partial V_{n+1}\left(x_{n+1}^{*}\right)$, where $p_{n+1}^{a}$ is the absolutely continuous part of $p_{n+1}$. To this end, note that from the definition of the concave subdifferential we have

$$
V_{n+1}(w)-V_{n+1}\left(x_{n+1}^{*}\right) \leq p_{n+1}\left(w-x_{n+1}^{*}\right) \quad \text { for all } w \in L^{\infty}\left(\Sigma_{n+1}, X\right) .
$$


Let $\left\{B_{m}\right\}_{m \geq 1} \subseteq \Sigma_{n+1}$ be the decreasing sequence of $\Sigma_{n+1}$-sets, which supports the singular part $p_{n+1}^{s}$ of $p_{n+1} \quad($ see $\S 2)$. Set

$$
w_{m}=\chi_{B_{m}^{c}} w+\chi_{B_{m}} x_{n+1}^{*} \in L^{\infty}\left(\Sigma_{n+1}, X\right) .
$$

Then using Proposition 2.1, we have for all $m \geq 1$

$$
\begin{aligned}
V_{n+1}\left(w_{m}\right)-V_{n+1}\left(x_{n+1}^{*}\right) & \leq\left\langle p_{n+1}^{a}, w_{m}-x_{n+1}^{*}\right\rangle+p_{n+1}^{s}\left(w_{m}-x_{n+1}^{*}\right) \\
& =\left\langle p_{n+1}^{a}, w_{m}-x_{n+1}^{*}\right\rangle+p_{n+1}^{s}\left(\chi_{B_{m}}\left(w_{m}-x_{n+1}^{*}\right)\right) \\
& =\left\langle p_{n+1}^{a}, w_{m}-x_{n+1}^{*}\right\rangle .
\end{aligned}
$$

Observe that $w_{m} \stackrel{\mu}{\rightarrow} w$ as $n \rightarrow \infty$, so by Proposition 2.2 we have that $\left\langle p_{n+1}^{a}, w_{m}-x_{n+1}^{*}\right\rangle \rightarrow\left\langle p_{n+1}^{a}, w-x_{n+1}^{*}\right\rangle$ as $m \rightarrow \infty$. We will also show that $V_{n+1}(w) \leq \varliminf_{m \rightarrow \infty} V_{n+1}\left(w_{m}\right)$. To this end, given $\varepsilon>0$, let $y \in C_{n+1}(w)$ such that

$$
V_{n+1}(w)-\varepsilon \leq \sum_{k=n+1}^{\infty} \delta^{k+1} J_{k+1}\left(y_{k}, y_{k+1}\right) .
$$

Let $y_{k}^{m}=\chi_{B_{m}^{c}} y_{k}+\chi_{B_{m}} x_{k}^{*} \in L^{\infty}\left(\Sigma_{k}, X\right)$ and $y_{k+1}^{m}=\chi_{B_{m}^{c}} y_{k+1}+\chi_{B_{m}} x_{k+1}^{*} \in$ $L^{\infty}\left(\Sigma_{k+1}, X\right)$. Then $y_{k}^{m} \stackrel{\mu}{\rightarrow} y_{k}$ and $y_{k+1}^{m} \stackrel{\mu}{\rightarrow} y_{k+1}$ as $m \rightarrow \infty$. So using hypothesis $H(u)_{1}$, we see that we can find $m_{0} \geq 1$ such that for $m \geq m_{0}$ we have

$$
\left|\sum_{k=n+1}^{\infty} \delta^{k+1} J_{k+1}\left(y_{k}^{m}, y_{k+1}^{m}\right)-\sum_{k=n+1}^{\infty} \delta^{k+1} J_{k+1}\left(y_{k}, y_{k+1}\right)\right|<\frac{\varepsilon}{2} .
$$

Hence we have for $m \geq m_{0}$

$$
\begin{aligned}
& V_{n+1}(w)-\frac{\varepsilon}{2} \leq \sum_{k=n+1}^{\infty} \delta^{k+1} J_{k+1}\left(y_{k}^{m}, y_{k+1}^{m}\right) \leq V_{n+1}\left(w_{m}\right) \\
& \Rightarrow V_{n+1}(w) \leq \varliminf_{m \rightarrow \infty} V_{n+1}\left(w_{m}\right) .
\end{aligned}
$$

So finally we get

$$
\begin{aligned}
& V_{n+1}(w)-V_{n+1}\left(x_{n+1}^{*}\right) \leq\left\langle p_{n+1}^{a}, w-x_{n+1}^{*}\right\rangle \quad \text { for all } w \in L^{\infty}\left(\Sigma_{n+1}, X\right) \\
& \quad \Rightarrow p_{n+1}^{a} \in \partial V_{n+1}\left(x_{n+1}^{*}\right) .
\end{aligned}
$$

In a similar fashion we can show that

$$
\left(z_{n}, z_{n+1}^{a}\right) \in \partial J_{n+1}\left(x_{n}^{*}, x_{n+1}^{*}\right) \text { and }\left(y_{n}, y_{n+1}^{a}\right) \in \partial \delta_{S_{n+1}}\left(x_{n}^{*}, x_{n+1}^{*}\right) .
$$

Note that since $-p_{n+1}=\delta^{n+1} z_{n+1}+y_{n+1}$ and using Proposition 2.1, we have

$$
-p_{n+1}^{a}=\delta^{n+1} z_{n+1}^{a}+y_{n+1}^{a} \text {. }
$$

Therefore, so far we have $p_{n} \in \partial V_{n}\left(x_{n}^{*}\right)$ and

$$
\begin{gathered}
\left(z_{n}, z_{n+1}^{a}\right) \in \partial J_{n+1}\left(x_{n}^{*}, x_{n+1}^{*}\right), \\
p_{n+1}^{a} \in \partial V_{n+1}\left(x_{n+1}^{*}\right), \\
\left(y_{n}, y_{n+1}^{a}\right) \in \partial \delta_{S_{n+1}}\left(x_{n}^{*}, x_{n+1}^{*}\right)
\end{gathered}
$$


with $p_{n}=\delta^{n+1} z_{n}+y_{n}$ and $-p_{n+1}^{a}=\delta^{n+1} z_{n+1}^{a}+y_{n+1}^{a}$. From (1) we get

$$
\begin{aligned}
\delta^{n+1} J_{n+1} & (f, g)-\delta^{n+1} J_{n+1}\left(x_{n}^{*}, x_{n+1}^{*}\right) \\
& \leq \delta^{n+1} z_{n}\left(v-x_{n}^{*}\right)+\left\langle\delta^{n+1} z_{n+1}^{a}, w-x_{n+1}^{*}\right\rangle \\
\Rightarrow & \delta^{n+1} J_{n+1}(f, g)-\delta^{n+1} z_{n}(v)-\left\langle\delta^{n+1} z_{n+1}^{a}, w\right\rangle \\
& \leq \delta^{n+1} J_{n+1}\left(x_{n}^{*}, x_{n+1}^{*}\right)-\delta^{n+1} z_{n}\left(x_{n}^{*}\right)-\left\langle\delta^{n+1} z_{n+1}^{a}, x_{n+1}^{*}\right\rangle
\end{aligned}
$$

for all $(f, g) \in L^{\infty}\left(\Sigma_{n}, X\right) \times L^{\infty}\left(\Sigma_{n+1}, X\right)$. Also from relation (3) we have

$$
0 \leq y_{n}\left(f-x_{n}^{*}\right)+\left\langle y_{n+1}^{a}, g-x_{n+1}^{*}\right\rangle
$$

for all $(f, g) \in S_{n+1}$. Adding inequalities (4) and (5) and recalling that $p_{n}=$ $\delta^{n+1} z_{n}+y_{n}$ and $-p_{n+1}^{a}=\delta^{n+1} z_{n+1}^{a}+y_{n+1}^{a}$, we get

$$
\begin{aligned}
& \delta^{n+1} J_{n+1}(f, g)-p_{n}(f)+\left\langle p_{n+1}^{a}, g\right\rangle \\
& \quad \leq \delta^{n+1} J_{n+1}\left(x_{n}^{*}, x_{n+1}^{*}\right)-p_{n}\left(x_{n}^{*}\right)+\left\langle p_{n+1}^{a}, x_{n+1}^{*}\right\rangle
\end{aligned}
$$

for all $(f, g) \in S_{n+1}$. We claim that the above inequality remains valid if we replace $p_{n} \in L^{\infty}\left(\Sigma_{n}, X\right)^{*}$ by its absolutely continuous part $p_{n}^{a} \in L^{1}\left(\Sigma_{n}, X^{*}\right)$. So as before let $\left\{B_{m}^{\prime}\right\}_{m \geq 1} \subseteq \Sigma_{n}$ be the decreasing sequence of $\Sigma_{n}$-sets supporting $p_{n}^{s}$. Define

$$
f_{m}=\chi_{B_{m}^{\prime}} f+\chi_{B_{m}^{\prime}} x_{n}^{*} \in L^{\infty}\left(\Sigma_{n}, X\right)
$$

and

$$
g_{m}=\chi_{B_{m}^{\prime} c} g+\chi_{B_{m}^{\prime}} x_{n+1}^{*} \in L^{\infty}\left(\Sigma_{n+1}, X\right)
$$

Note that

$$
\begin{aligned}
p_{n}\left(f_{m}-x_{n}^{*}\right) & =\left\langle p_{n}^{a}, f_{m}-x_{n}^{*}\right\rangle+p_{n}^{s}\left(f_{m}-x_{n}^{*}\right) \\
& =\left\langle p_{n}^{a}, f_{m}-x_{n}^{*}\right\rangle+p_{n}^{s}\left(\chi_{B_{m}^{\prime}}\left(f_{m}-x_{n}^{*}\right)\right)=\left\langle p_{n}^{a}, f_{m}-x_{n}^{*}\right\rangle \\
& \rightarrow\left\langle p_{n}^{a}, f-x_{n}^{*}\right\rangle \quad \text { as } m \rightarrow \infty,
\end{aligned}
$$

since $f_{m} \stackrel{\mu}{\rightarrow} f$, so, by Proposition 2.2, $f_{m} \stackrel{\tau}{\rightarrow} f$ in $L^{\infty}\left(\Sigma_{n}, X\right)$. Since $J_{n+1}\left(f_{m}, g_{m}\right) \rightarrow J_{n+1}(f, g)$ in the limit as $m \rightarrow \infty$, we get from (6) that

$$
\begin{aligned}
& \delta^{n+1} J_{n+1}(f, g)-\left\langle p_{n}^{a}, f\right\rangle+\left\langle p_{n+1}^{a}, g\right\rangle \\
& \quad \leq \delta^{n+1} J_{n+1}\left(x_{n}^{*}, x_{n+1}^{*}\right)-\left\langle p_{n}^{a}, x_{n}^{*}\right\rangle+\left\langle p_{n+1}^{a}, x_{n+1}^{*}\right\rangle .
\end{aligned}
$$

Furthermore, as we did for $p_{n+1}$, we can show that $p_{n}^{a} \in \partial V_{n}\left(x_{n}^{*}\right)$. Hence we have established that if $p_{n} \in \partial V_{n}\left(x_{n}^{*}\right)$, then $p_{n}^{a} \in \partial V_{n}\left(x_{n}^{*}\right)$, and we can also produce $p_{n+1}^{a} \in L^{1}\left(\Sigma_{n+1}, X^{*}\right)$ such that $p_{n+1}^{a} \in \partial V_{n+1}\left(x_{n+1}^{*}\right)$ and $\delta^{n+1} J_{n+1}(f, g)-$ $\left\langle p_{n}^{a}, f\right\rangle+\left\langle p_{n+1}^{a}, g\right\rangle \leq \delta^{n+1} J_{n+1}\left(x_{n}^{*}, x_{n+1}^{*}\right)-\left\langle p_{n}^{a}, x_{n}^{*}\right\rangle+\left\langle p_{n+1}^{a}, x_{n+1}^{*}\right\rangle$ for all $(f, g)$ $\in S_{n+1}$. From hypothesis $H_{0}$ we know that $\partial V_{0}\left(\bar{x}_{0}\right) \neq \varnothing$. So by induction we can produce a sequence of functions $p_{n} \in L^{1}\left(\Sigma_{n}, X^{*}\right)$ which satisfy inequalities (i) and (ii) in the conclusion of the theorem. We will show that, for every $n \geq 1, p_{n} \geq 0$. Indeed let $e \in L^{\infty}\left(\Sigma_{n}, X\right)_{+}$. Set $z_{n}=x_{n}^{*}+e$. Then from the free disposability hypothesis we have $\left(z_{n}, x_{n+1}^{*}\right) \in S_{n+1}$. Hence we have

$$
\begin{aligned}
\delta^{n+1} J_{n+1}\left(z_{n}, x_{n+1}^{*}\right)-\left\langle p_{n}^{a}, z_{n}\right\rangle+\left\langle p_{n+1}^{a}, x_{n+1}^{*}\right\rangle \\
\leq \delta^{n+1} J_{n+1}\left(x_{n}^{*}, x_{n+1}^{*}\right)-\left\langle p_{n}^{a}, x_{n}^{*}\right\rangle+\left\langle p_{n+1}^{a}, x_{n+1}^{*}\right\rangle \\
\Rightarrow \delta^{n+1}\left(J_{n+1}\left(z_{n}, x_{n+1}^{*}\right)-J_{n+1}\left(x_{n}^{*}, x_{n+1}^{*}\right)\right) \leq\left\langle p_{n}^{a}, e\right\rangle .
\end{aligned}
$$


Because of hypothesis $H(u)_{1}$, we have

$$
\begin{aligned}
0 & \leq J_{n+1}\left(z_{n}, x_{n+1}^{*}\right)-J_{n+1}\left(x_{n}^{*}, x_{n+1}^{*}\right) \\
& \Rightarrow 0 \leq\left\langle p_{n}^{a}, e\right\rangle \quad \text { for all } e \in L^{\infty}\left(\Sigma_{n}, X\right)_{+} \\
& \Rightarrow p_{n}^{a} \geq 0 \quad \text { for all } n \geq 0 .
\end{aligned}
$$

Finally for the transversality condition (iii) note that

$$
\begin{aligned}
& V_{n}(0)-\left\langle p_{n}, 0\right\rangle \leq V_{n}\left(x_{n}^{*}\right)-\left\langle p_{n}, x_{n}^{*}\right\rangle \\
& \quad \Rightarrow 0 \leq\left\langle p_{n}, x_{n}^{*}\right\rangle \leq V_{n}\left(x_{n}^{*}\right)-V_{n}(0) .
\end{aligned}
$$

Because of hypothesis $H(u)_{1}$, we have $\lim _{n \rightarrow \infty}\left(V_{n}\left(x_{n}^{*}\right)-V_{n}(0)\right)=0$, so

$$
\lim _{n \rightarrow \infty}\left\langle p_{n}, x_{n}^{*}\right\rangle=0 \text {. Q.E.D. }
$$

Remarks. (a) Note that if $(f, g) \in S_{n+1},\left\langle p_{n}, f\right\rangle$ is the cost of the input $f$, $\left\langle p_{n+1}, g\right\rangle$ is the value of the output $g$, so $\left\langle p_{n+1}, g\right\rangle-\left\langle p_{n}, f\right\rangle$ is the profit resulting from operating technological process $(f, g) \in S_{n+1}$ at the $(n+1)$ stage. Also, $\delta^{n+1} J_{n+1}(f, g)$ is the discounted utility corresponding to $(f, g)$. Hence $\delta^{n+1} J_{n+1}(f, g)-\left\langle p_{n}, f\right\rangle+\left\langle p_{n}, g\right\rangle$ is the total utility generated by process $(f, g)$. Then inequality (ii) of Theorem 4.1 tells us that along an optimal program we have maximization of the total utility among all other feasible programs.

(b) Inequality (i) in Theorem 4.1 says that along an optimal program we have minimization of the cost among all other programs producing no less future value.

(c) The transversality condition (iii) tells us that the expected value of the input (and output) at time $n$ goes to zero as $n \rightarrow \infty$.

(d) A program $\hat{x}^{*} \in F\left(\bar{x}_{0}\right)$ and a price system $\hat{p}=\left\{p_{n}\right\}_{n \geq 0}$ satisfying inequality (ii) of the theorem form a pair $[\hat{x}, \hat{p}]$ which is called "competitive" (see Takayama [10]). So according to Theorem 4.1, every optimal program $\hat{x}^{*}$ admits a price system $\hat{p}=\left\{p_{n}\right\}_{n \geq 0}$ so that the pair $\left[\hat{x}^{*}, \hat{p}\right]$ is competitive.

Next we prove the converse of Theorem 4.1; namely, we show that if a pair $[\hat{x}, \hat{p}]$ is competitive (see Remark (d)) and satisfies the transversality condition, then $\hat{x}$ is optimal.

Theorem 4.2. If hypotheses $H(P)$ and $H(u)_{1}$ hold, $\left[\hat{x}^{*}, \hat{p}\right]$ is a competitive pair, and $\lim _{n \rightarrow \infty}\left\langle p_{n}, x_{n}^{*}\right\rangle=0$, then $\hat{x}^{*}$ is an optimal program.

Proof. Since by hypothesis $\left[\hat{x}^{*}, \hat{p}\right]$ is competitive, we have for all $\hat{y} \in F\left(\bar{x}_{0}\right)$ that

$$
\begin{aligned}
& \sum_{k=0}^{N} \delta^{k+1}\left(J_{k+1}\left(y_{k}, y_{k+1}\right)-J_{k+1}\left(x_{k}^{*}, x_{k+1}^{*}\right)\right) \\
& \quad \leq \sum_{k=0}^{N}\left(p_{k+1}, x_{k+1}^{*}-y_{k+1}\right\rangle+\left\langle p_{k}, y_{k}-x_{k}^{*}\right\rangle \leq\left\langle p_{N+1}, x_{N+1}^{*}-y_{N+1}\right\rangle \\
& \quad \leq\left\langle p_{N+1}, x_{N+1}^{*}\right\rangle \quad \text { since } p_{N+1} \geq 0, y_{N+1} \geq 0 .
\end{aligned}
$$

Hence passing to the limit as $N \rightarrow \infty$ and since by hypothesis $\left\langle p_{N+1}, x_{n+1}^{*}\right\rangle$ 
$\rightarrow 0$ as $N \rightarrow \infty$, we get

$$
\begin{aligned}
& \sum_{k=0}^{\infty} \delta^{k+1} J_{k+1}\left(y_{k}, y_{k+1}\right) \leq \sum_{k=0}^{\infty} \delta^{k+1} J_{k+1}\left(x_{k}^{*}, x_{k+1}^{*}\right) \\
& \Rightarrow U(\hat{y}) \leq U\left(\hat{x}^{*}\right) .
\end{aligned}
$$

Since $\hat{y} \in F\left(\bar{x}_{0}\right)$ was arbitrary, we conclude that $\hat{x}^{*}$ is indeed optimal. Q.E.D.

\section{ACKNOWLEDGMENT}

The author expresses his gratitude to the anonymous referee for constructive criticisms and for bringing to his attention some additional references. The author also expresses his gratitude to Professor J. S. B. Mitchell, the editor, for his very kind and encouraging attitude.

\section{REFERENCES}

1. C. Aliprantis, D. Brown, and O. Burkinshaw, Existence and optimality of competitive equilibria, Springer, Berlin, 1988.

2. K. Arrow and M. Kurz, Public investment, the rate of return and optimal fiscal policy, Johns Hopkins Press, Baltimore, 1970.

3. H. Attouch, Variational convergence for functionals and operators, Pitman, London, 1984.

4. G. Buttazzo, Semicontinuity, relaxation and integral representation in the calculus of variations, Pitman Res. Notes Math. Ser., vol. 207, Longman Sci. Tech., Harlow, 1989.

5. J. Diestel and J. Uhl, Vector measures, Math. Surveys Monographs, vol. 15, Amer. Math. Soc., Providence, RI, 1977.

6. M. Dolcher, Topologie e strutture di convergenza, Ann. Scuola Norm. Sup. Pisa Cl. Sci. (4) 14 (1960), 63-92.

7. A. Ioffe and V. Tichomirov, Theory of extremal problems, North-Holland, Amsterdam, 1979.

8. V. Levin, The Lebesgue decomposition for functionals on the vector function space $L^{\infty}(X)$, Functional Anal. Appl. 8 (1974), 314-317.

9. B. Peleg and H. Ryder, On optimal consumption plans in a multisector economy, Rev. Econom. Stud. 39 (1972), 159-169.

10. A. Takayama, Mathematical economics, second edition, Cambridge Univ. Press, Cambridge, England, 1985.

11. M. L. Weitzman, Duality theory for infinite horizon convex models, Management Sci. 19 (1973), 783-789.

12. K. Yosida and E. Hewitt, Finitely additive measures, Trans. Amer. Math. Soc. 72 (1952), 45-66.

13. I. Zilha, Characterization by prices of optimal programs under uncertainty, J. Math. Econom. 3 (1976), 173-183.

14. V. Arkin and I. V. Evstigneev, Stochastic models of control and economic dynamics, Academic Press, London, 1987.

15. E. B. Dynkin, Some probability models for a developing economy, Soviet Math. Dokl. 12 (1971), 1422-1425.

16. _ Concave stochastic dynamic programming, Math. USSR-Sb. 16 (1972), 501-515.

17. I. V. Evstigneev, Optimal stochastic programs and their stimulating prices, Mathematical Models in Economics (J. Los and M. Los, eds.), North-Holland, Amsterdam, 1974, pp. 219-252. 
18. R. Radner, Optimal stationary consumption with stochastic production and resources, J. Econom. Theory 6 (1973), 68-90.

19. M. I. Taksar, Optimal planning over infinite time interval under random factors, Mathematical Methods in Economics (J. Los and M. Los, eds.), North-Holland, Amsterdam, 1974, pp. 284-298.

National Technical University, Department of Mathematics, Zografou Campus, ATHENS 15773, GREECE

Florida Institute of Technology, Department of Applied Mathematics, 150 West UniVERsity Boulevard, MELBOURNe, Florida 32901-6988 